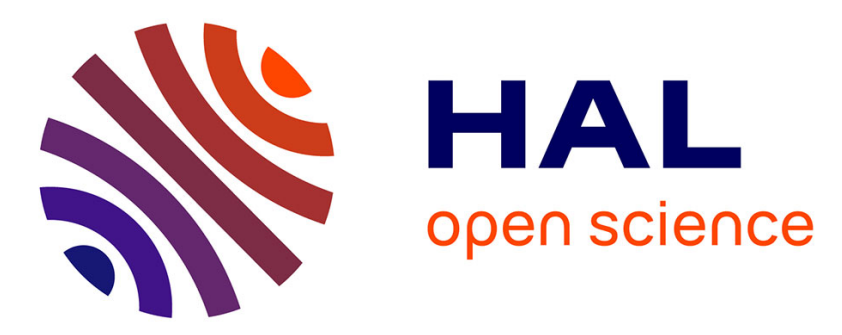

\title{
Lateral quantization effects in the optical properties of barrier modulated InGaAs/GaAs wires
}

Ch. Gréus, R. Spiegel, F. Faller, A. Forchel

\section{To cite this version:}

Ch. Gréus, R. Spiegel, F. Faller, A. Forchel. Lateral quantization effects in the optical properties of barrier modulated InGaAs/GaAs wires. Journal de Physique IV Proceedings, 1993, 03 (C5), pp.C5139-C5-142. 10.1051/.jp4:1993524 . jpa-00251610

\section{HAL Id: jpa-00251610 https://hal.science/jpa-00251610}

Submitted on 1 Jan 1993

HAL is a multi-disciplinary open access archive for the deposit and dissemination of scientific research documents, whether they are published or not. The documents may come from teaching and research institutions in France or abroad, or from public or private research centers.
L'archive ouverte pluridisciplinaire HAL, est destinée au dépôt et à la diffusion de documents scientifiques de niveau recherche, publiés ou non, émanant des établissements d'enseignement et de recherche français ou étrangers, des laboratoires publics ou privés. 


\title{
Lateral quantization effects in the optical properties of barrier modulated InGaAs/GaAs wires
}

\author{
Ch. GRÉUS, R. SPIEGEL, F. FALLER and A. FORCHEL \\ Technische Physik, Universität Würzburg, Am Hubland, 97074 Würzburg, Germany
}

\begin{abstract}
Abstact: We have studied the optical properties of barrier modulated $\mathrm{InGaAs} / \mathrm{GaAs}$ quantum wires with widths down to about $20 \mathrm{~nm}$. Here the lateral confinement is introduced via the change of the band discontinuity due to the modulation of the upper barrier of a quantum well structure. For wire widths below $50 \mathrm{~nm}$ the emission spectra shift systematically to higher energy which is associated to the lateral confinement. Using the geometrical wire widths measured by scanning electron microscopy the width dependence of the emission can be calculated in good agreement with the experimental data. In photoluminescence excitation experiments up to three transitions which arise from lateral confined states can be observed. For the smallest wires we observe a broadening of the emission spectra which can be explained in terms of size fluctuation. The barrier modulated wires show a strong linear polarization of the emission parallel to the wire axis up to about $25 \%$ for $20 \mathrm{~nm}$ wide wires.
\end{abstract}

\section{INTRODUCTION}

Quasi-one-dimensional and zero-dimensional structures are of great interest both for studying the basic physical properties and for possible device applications. For such systems different new phenomena have been predicted depending strongly on the lateral size of these structures. To realize quantum wires and quantum dots several approaches have been used and met with different degrees of success. [1-4]

In this paper we have used the barrier modulation technique for the fabrication of effectively buried wires. Using a selective etchant the upper barrier of an InGaAs/GaAs quantum well structure is modulated. Due to the change of the energy band discontinuity a lateral potential is introduced leading to a confinement of the carriers below the remaining GaAs stripes.[5] The wires show high quantum efficiency for all wire widths indicating that nonradiative recombination of carriers in the wires plays no significant role. Decreasing the wire width below $50 \mathrm{~nm}$ the transition energy shifts significantly to higher values and we observe in photoluminescence excitation spectra additional structures associated with higher lateral subbands. All transitions can be described by a simple model using the geometrical wire width. In addition we observe a strong polarization of the wire emission parallel to the wire axis.

\section{CONCEPT AND TECHNOLOGY OF BARRIER MODULATED WIRES}

In the concept of lateral barrier modulation the basic idea is the introduction of a lateral confinement potential within the active layer via the modulation of the upper barrier. Using a selective etchant the material of the upper barrier (GaAs) of an InGaAs/GaAs quantum well structure is locally removed whereas the active InGaAs layer remains over the whole plane. Compared to the semiconductor covered quantum well the quantization energy of a surface quantum well is significantly increased due to the replacement of the InGaAs/GaAs transition by the InGaAs/Vacuum transition in 
the etched regions. The corresponding lateral potential for both electrons and holes leads to a confinement of the carriers below the remaining GaAs stripes. Due to the selectivity of the etchant the process provides effectively buried wires and the active area is not affected by process induced defects.

The starting material for the wire fabrication are $\mathrm{In}_{\mathrm{x}} \mathrm{Ga}_{1-\mathrm{x}} \mathrm{As} / \mathrm{GaAs}$ single quantum wells grown by molecular beam epitaxy on (100) oriented undoped GaAs substrates. Different samples with In contents between $9 \%$ and $18 \%$ were used. The InGaAs layer which acts as the active layer has a thickness of $5 \mathrm{~nm}$ for all samples and is covered by a thin GaAs cap layer (thickness $15 \mathrm{~nm}-20 \mathrm{~nm}$ ). As can be shown by a calculation of the energy levels of the quantum well structures only one electron level as well as one heavy-hole and one light-hole level are bound. Due to the lattice mismatch between $\mathrm{GaAs}$ and $\mathrm{In}_{\mathrm{x}} \mathrm{Ga}_{1-\mathrm{x}}$ As the light-hole level is shifted far away from the edge of the valence band. As a consequence of the large splitting between the light- and heavy-hole levels only a negligible intermixing of the valence band states is expected for these structures.

Using high resolution electron beam lithography and $\mathrm{Al}$ lift off, mask patterns with typical dimensions of $100 \mu \mathrm{m}$ in square were defined. Each pattern consists of an array of parallel wires with constant width. The patterns were transferred to the semiconductor by a single wet etch step with the selective etchant $\mathrm{H}_{2} \mathrm{O}_{2}$ buffered with $\mathrm{NH}_{4} \mathrm{OH}$. [6] In that way barrier modulated wires with widths from several microns down to about $20 \mathrm{~nm}$ were defined. The measurements were performed using an Ar laser with typical excitation power of $1 \mathrm{~mW}$. The emission spectra were energetically dispersed by a $0.25 \mathrm{~m}$ grating monochromator. For the detection a multichannel analyzer with $\mathbf{S} 25$ characteristic was used. For the photoluminescence excitation experiments a titan-sapphire laser was used as excitation source. The signal was recorded by a photomultiplier.

\section{EXPERIMENTAL RESULTS AND DISCUSSION}

In order to study the optical properties of the barrier modulated wires photoluminescence experiments were performed at a temperature of $2 \mathrm{~K}$. All wires show high quantum efficiency even for wire widths smaller than $30 \mathrm{~nm}$. For the smallest wires with geometrical width of about $20 \mathrm{~nm}$ the normalized emission intensity is reduced by only a factor of 5 with respect to the unprocessed asgrown 2D reference.

In Figure 1 the increase of the emission energy compared to the $2 \mathrm{D}$ as-grown reference is plotted as a function of the wire width for barrier modulated wires defined on an $\mathrm{In}_{0.09} \mathrm{Ga}_{0.91}$ As quantum well structure (filled triangle). The wire widths given in Fig. 1 are the geometrical widths as measured by scanning electron microscopy. The position of the emission peak remains almost unchanged for wire widths down to about $50 \mathrm{~nm}$. For wider wires we observe a slight shift of the emission peak to lower energy. This may be attributed to small changes of the strain in the InGaAs layer due to the fabrication process.

For wires with widths below $50 \mathrm{~nm}$ a strong shift of the transition energy can be observed with decreasing wire width which amounts to about $7 \mathrm{meV}$ for the $20 \mathrm{~nm}$ wide wires. This blue shift is connected with the lateral confinement. The steep increase of the transition energy indicates that a sharp potential has been obtained by the modulation of the upper barrier material leading to onedimensional quantum effects for wires with widths below $50 \mathrm{~nm}$.

We have calculated the electronic states of the wires using the standard material parameters. The Schrödinger equation was solved separately for the two confinement directions in the effective-mass approximation. We assume a lateral square well potential for both electrons and holes which is determined by the difference between the quantization energy for the $\mathrm{GaAs} / \mathrm{InGaAs} /$ Vacuum

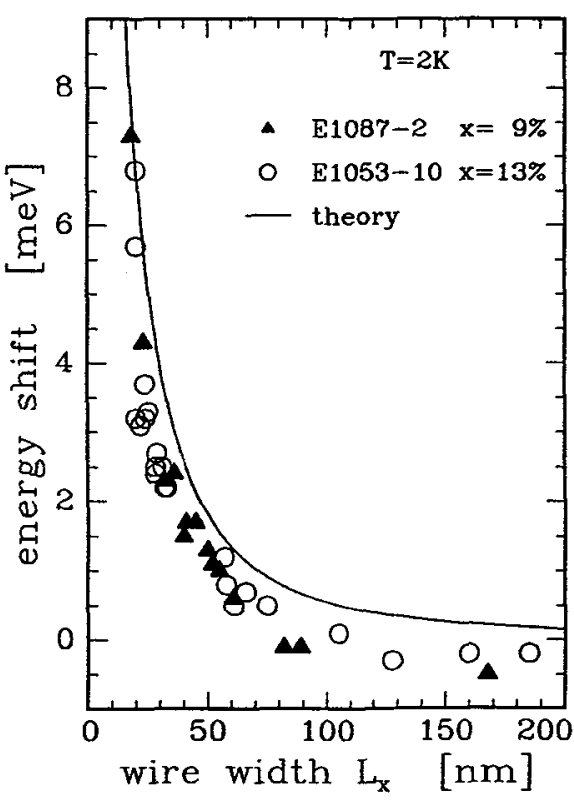

Fig. 1: Wire width dependence of the energy shift for the lowest subband transition. Symbols: experiment, solid line: results of the calculation described in the text. 
surface quantum well and the GaAs/InGaAs/GaAs quantum well of the starting structure. $[5,6]$

The solide curve in Fig. 1 represents the results of the calculated energy shifts for the lowest lying transition for the barrier modulated $\mathrm{In}_{0.09} \mathrm{Ga}_{0.91}$ As wires. Using the geometrical wire width the calculation yields good agreement with the experimental data without any fitable parameter. The constant difference of about $0.5 \mathrm{meV}$ can be explained with the slight shift to lower energy observed for the wider wires.

We have made similar experiments for barrier modulated wires on samples with varying In content of the active layer. For an In content of $13 \%$ the experimental energetic shifts are shown by the open circles in Fig. 1 for different wire widths. Due to the higher In content the emission peak shifts to lower energy, but the wire widths dependence of the blue shift of the transition energy has the same slope and onset for both samples. This is consistent with model calculations. For both systems the lateral potential which arises from the modulation of the upper barrier is in the range of about $23 \mathrm{meV}$ (electrons and holes). Therefore it is expected that the lowest lying wire states show a nearly identical dependence on the wire width for both samples. This is in good agreement with the experiment indicating that the energetic levels are determined predominantly by the lateral potential whereas strain effects play a minor role.

The Full Width at Half Maximum (FWHM) of the photoluminescence emission remains equal to the value of the reference for wire widths down to about $50 \mathrm{~nm}$. For smaller wires the FWHM increases with decreasing wire width due to the stronger influence of size fluctuations for a reduced lateral size. Although the spectral line width of the two reference samples are different, the additional broadening shows the same dependence on the wire width. We attribute this broadening to fluctuations of the lateral dimension of the wires. Assuming a lateral size fluctuation $\Delta \mathrm{L}_{\mathrm{x}}$ of $\pm 10 \mathrm{~nm}$, which is consistent with estimates based on SEM measurements, the calculation of the additional broadening of the emission peak yields good agreement with the experimentally observed data.

Photoluminescence excitation (PLE) experiments have been performed to study the higher lying states of the quantum wires. Figure 2 displays the PLE spectrum for $52 \mathrm{~nm}$ wide wires detected at $847 \mathrm{~nm}$. In addition, the photoluminescence intensity (PL) is shown by the dashed line in Fig. 2 . The observed stokes-shift amounts to about $1 \mathrm{meV}$ indicating the high quality of the barrier modulated wire structure. The most important aspect in Fig. 2 is the appearence of two features in the PLE spectrum which occures at higher energies (arrows at $1.472 \mathrm{eV}$ and $1.480 \mathrm{eV}$ ). Studies on samples with different wire width show that the energetic difference between these peaks increases for decreasing wire width.

Therefore we attribute these features to transitions associated with higher lying states in the wires. Based on the geometrical width of the wires we calculate the energetic levels arising from the lateral confinement. The results of the calculation for the $52 \mathrm{~nm}$ wires are shown by the arrows in Fig. 2 for the $e_{1,1}-h_{1,1}, e_{1,2}-h_{1,2}$ and $\mathrm{e}_{1,3}-\mathrm{hh}_{1,3}$ transitions. Here the first number indicates the vertical quantum number whereas the second number denotes the lateral quantum number. The overall good agreement with the experimentally observed data confirms that these peaks corresponds to lateral bound states.

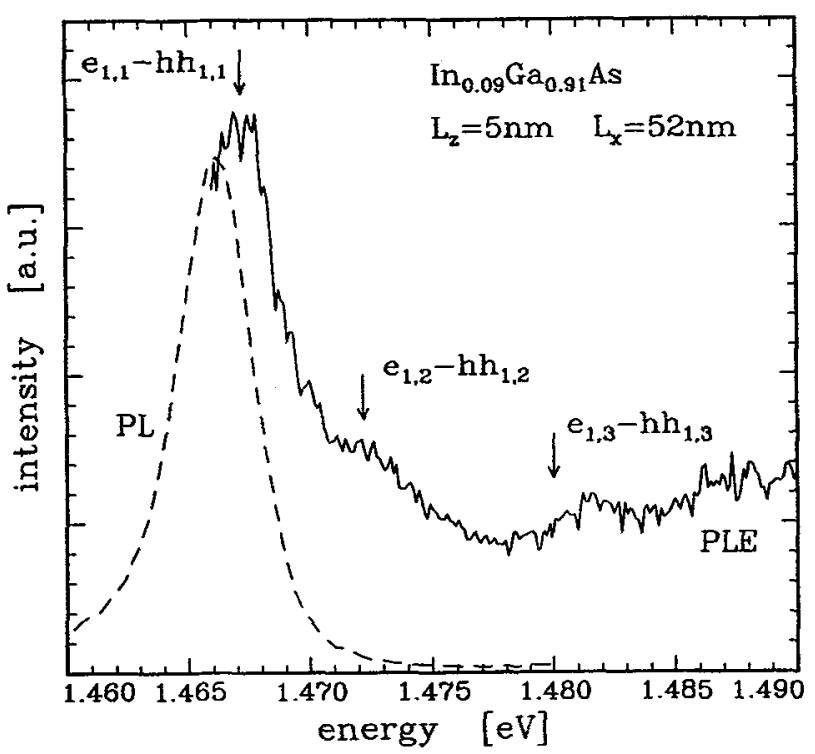

Fig. 2: Photoluminescence and photoluminescence excitation spectra for $52 \mathrm{~nm}$ wide wires. The arrows indicate the calculated subband transitions. 
We have investigated photoluminescence spectra for different linear polarization orientations. The wire emission shows a clear polarization parallel to the wire axis. Figure 3 shows the experimental data of the polarization degree of the emission from barrier modulated wires defined on $\mathrm{In}_{0.09} \mathrm{Ga}_{0.91} \mathrm{As}$. Here, $\mathrm{I}_{\|}\left(\mathrm{I}_{1}\right)$ denotes the emission intensity parallel (perpendicular) to the wire axis.

The polarization degree increases with decreasing wire width up to about $25 \%$ for the smallest wires. The linear polarization may be due to different sources. Theoretical calculations predict polarized emission due to a mixing of the light- and heavy-hole states in the wires [8]. Furthermore electrodynamic effects can cause a polarization of the wire emission [9]. As the experiments were performed on arrays of parallel wires the modulation of the surface acts as a grating structure. Due to the periodic variation of the dielectric constant parallel to the surface the local electric field which is induced by the incoming light depends on the polarization of the beam [9]. In particular, this effect may be the reason for the high polarization degree observed for wires in the range of 1000 $-100 \mathrm{~nm}$.

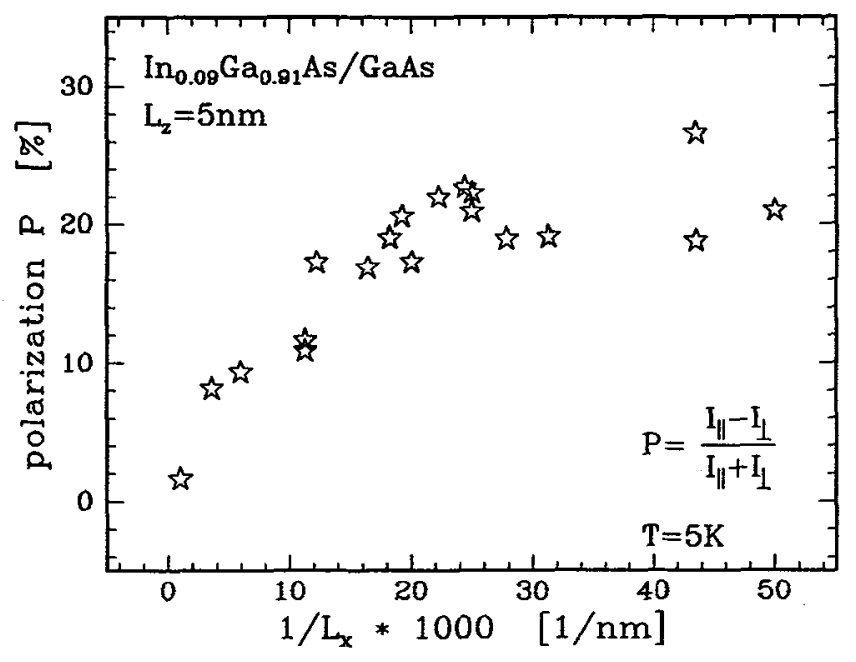

Fig. 3: Degree of polarization of the wire emission parallel to the wires versus the wire width $L_{x}$.

In conclusion, we have fabricated high quality barrier modulated $\mathrm{InGaAs} / \mathrm{GaAs}$ wires with lateral dimensions down to $20 \mathrm{~nm}$. It is found that the emission of the $e_{1,1}-\mathrm{hh} h_{1,1}$ transition is unchanged for wire widths down to $50 \mathrm{~nm}$. The significant increase of the emission energy for smaller wires is determined by the lateral potential. PLE experiments show clearly up to three lateral subband transitions. The good agreement between the experimental data and the results of simple model calculations demonstrates that the electronic properties of the wires can be understood quantitatively on the basis of the measured physical dimensions of the structure. The strong linear polarization of the wire emission is most likely mainly due to grating effects.

The financial support of this work by the Deutsche Forschungsgemeinschaft and the ESPRIT Basic Research Programme is gratefully acknowledged.

\section{REFERENCES}

1 K. Kash, A. Scherer, J. M. Worlock, H. G. Craighead, and M. C. Tamargo, Appl. Phys. Lett. 49, 1042 (1986).

2 J. Cibert, P. M.- Petroff, G. J. Dolan, S. J. Pearton, A. C. Gossard, and J. H. English, Appl. Phys. Lett. 49, 1275 (1986).

3 B. E. Maile, A. Forchel, R. Germann, J. Straka, L. Korte, and C. Thanner, Appl. Phys. Lett. 57, 807 (1990).

4 M. Notomi, M. Naganuma, T. Nishida, T. Tamamura, H. Iwamura, S. Nojima, and M. Chamoto, Appl. Phys. Lett. 58, 720 (1991).

5 Ch. Gréus, L. Butov, F. Daiminger, A. Forchel, P. A. Knipp, and T. L. Reinecke, Phys. Rev. Lett 47, 7626 (1993).

6 For GaAs the effective electron and hole masses were taken to be 0.066 and 0.341 , for $\mathrm{In}_{0.09} \mathrm{Ga}_{0.91}$ As to be 0.062 and 0.338 , and for $\mathrm{In}_{0.13} \mathrm{Ga}_{0.87} \mathrm{As}$ to be 0.061 and 0.336 . The band offset between $\mathrm{GaAs}$ and $\mathrm{In}_{\mathrm{x}} \mathrm{Ga}_{1-\mathrm{x}}$ As were taken to be 0.61 .

7 Ch. Gréus, A. Forchel, J. Straka, K. Pieger, and M. Emmerling, Appl. Phys. Lett 61, 1199 (1992).

8 U. Bockelmann and G. Bastard, Phys. Rev. B 45, 1688 (1992).

9 U. Bockelmann, Europhys. Lett. 16, 601 (1991). 